\title{
Live weight and sex effects on carcass and meat quality of "Borrego terrincho-PDO" suckling lambs
}

\author{
V.A.C. Santos *, S.R. Silva, E.G. Mena, J.M.T. Azevedo \\ CECAV - University of Trás-os-Montes and Alto Douro, Department of Animal Science, Apartado 1013, 5001-801 Vila Real, Portugal
}

Received 15 February 2007; received in revised form 11 May 2007; accepted 14 May 2007

\begin{abstract}
Fifty seven suckling lambs (28 males and 29 females) of the Churra da Terra Quente breed were used to evaluate the effects of live weight and sex on carcass composition and meat quality traits. Lambs were slaughtered at three weight classes $(<8 \mathrm{~kg}, 8-11 \mathrm{~kg}$ and $>11 \mathrm{~kg}$ ) according to "Borrego Terrincho-PDO" specifications. The left sides of the carcasses were totally dissected. The longissimus thoracis and lumborum muscle was used for meat quality determination. Dressing proportion and carcass fatness were not affected by weight class or sex. Muscle proportion was similar in all carcass joints for the three weight classes. The percentage of bone decreased at the higher weight class, while that of subcutaneous and intermuscular fat increased. Female lambs had higher muscle proportions and greater muscle/bone ratios in the carcass side than males. Muscle $\mathrm{pH}$, colour, cooking losses and tenderness were not affected by gender and weight class. Female lambs and weight classes $8-11 \mathrm{~kg}$ and $>11 \mathrm{~kg}$ had higher proportions of intramuscular fat.
\end{abstract}

(C) 2007 Elsevier Ltd. All rights reserved.

Keywords: Suckling lambs; Carcass composition; Meat quality; Slaughter weight

\section{Introduction}

A diversity of sheep breeds and production systems produce a large variety of carcass types in both the regional and European lamb market. One of the most widely used systems in sheep farming in Mediterranean countries involves the slaughter of lambs of dairy breeds at 25-45 days of age at a low body weight of $\approx 10-11 \mathrm{~kg}$ (Sañudo, Sanchez, \& Alfonso, 1998). The Churra da Terra Quente breed, from north-east Portugal, is mainly used for milk production and milk-fed lambs, which are slaughtered at 4-6 weeks of age, give carcasses of $\approx 4-8 \mathrm{~kg}$. Since 1996, the European Union has granted this meat a Protected Designation of Origin (PDO), "Borrego Terrincho" (Commission regulation EEC no. 1107/96). European meat quality labels, and in particular "Borrego Terrincho"-PDO, have some specificity attributed to a particular region and to a traditional production system. However, only a few studies on carcass

\footnotetext{
${ }^{*}$ Corresponding author. Tel.: +351 259350430; fax: +351 259350482. E-mail address: vsantos@utad.pt (V.A.C. Santos).
}

tissue distribution and meat quality traits of Portuguese local breeds have been published (Santos-Silva, Mendes, \& Bessa, 2002; Santos-Silva \& Portugal, 2001; Teixeira, Batista, Delfa, \& Cadavez, 2005). The carcass weight range in suckling lambs is small. Nevertheless, some differences in carcass tissue composition have been reported (Cañeque et al., 2004; Díaz et al., 2005; Díaz et al., 2003). As regards meat quality an increase in carcass weight of suckling lambs had a small influence on meat quality traits (Díaz et al., 2005; Russo, Preziuso, \& Verità, 2003) and no effect in sensorial properties (Sañudo, Santolaria, Maria, Osorio, \& Sierra, 1996). At early ages differences between male and female lambs carcasses are mainly reflected in the fatness parameters (Díaz et al., 2003; Horcada, Beriain, Purroy, Lizaso, \& Chasco, 1998; Velasco et al., 2000). In meat production systems a small increase in the slaughter weight of lambs may result in higher productivity. Borrego Terincho lambs are usually slaughtered at $8-11 \mathrm{~kg}$ of live weight, however in recent years there was a demand for lambs of lower weights. The aim of this study was to evaluate the effects of slaughter weight $(<8 \mathrm{~kg} ; 8-11 \mathrm{~kg} ;>11 \mathrm{~kg})$ and 
sex on carcass composition and meat quality of suckling lambs protected by the PDO "Borrego Terrincho".

\section{Material and methods}

\subsection{Animals}

The study was conducted with fifty seven suckling lambs ( 29 males and 28 females) of the native Portuguese breed Churra da Terra Quente, produced according to "Borrego Terrincho-PDO" specifications. Male and female lambs were randomly allocated to one of the three weight classes: $<8 \mathrm{~kg}(6.7 \pm 0.22 \mathrm{~kg}) ; 8-11 \mathrm{~kg}(9.8 \pm 0.20 \mathrm{~kg}) ;>11 \mathrm{~kg}$ $(12.7 \pm 0.43 \mathrm{~kg})$. Lambs were weighed weekly until they reached their target slaughter weight. Live weight at slaughter (SLW) was recorded after an overnight fast (Table 1) and lambs were slaughtered using standard commercial procedures.

\subsection{Carcass measurements, jointing and dissection}

Carcasses were refrigerated for $24 \mathrm{~h}$ at $4{ }^{\circ} \mathrm{C}$ and cold carcass weight $(\mathrm{CCW})$ and carcass dressing was recorded following the methods of Fisher and de Boer (1994). After the removal of the kidney knob and channel fat (KKCF), carcasses were split down the dorsal midline and the left sides were jointed into eight standardized commercial joints as outlined by Santos-Silva et al. (2002).

Carcass measurements included, leg length (from the symphysis pubis to the tarsal-metatarsal joint); internal carcass length ( $L$ : length from cranial edge of the symphysis pubis to the cranial edge of the first rib) and the perimeters (chest circumference, anterior buttock circumference and posterior buttock circumference). Carcass compactness, used as a conformation indicator, was determined as the ratio between $\mathrm{CCW}$ and internal carcass length $(\mathrm{CCW} / \mathrm{L} ; \mathrm{kg} / \mathrm{m})$. Dissection of the carcass joints was carried out following the Fisher and de Boer (1994) method, obtaining muscle, bone and fat. Subcutaneous, intermuscular and pelvic fat depots were weighed separately, in a dissection room with a controlled environment.

\subsection{Muscle sampling}

Samples of longissimus thoracis et lumborum (LTL) muscle were taken $24 \mathrm{~h}$ after slaughter, from the loin and rib joints, when carcass left halves were partitioned into commercial joints. One part of the muscle was vacuum packed and aged at $2 \pm 2{ }^{\circ} \mathrm{C}$ for $72 \mathrm{~h}$ after slaughter for cooking loss and tenderness determinations. The remaining portion, of $\approx 100 \mathrm{~g}$, was placed in a sealed plastic box, and stored at $-20{ }^{\circ} \mathrm{C}$ for later chemical analysis.

\subsection{Meat quality measurements}

A pH-meter equipped with a penetrating electrode and thermometer (Hanna Instruments, HI-9025) was used to determine $\mathrm{pH}$ in the LTL muscle (1st to 2 nd lumbar vertebra), $60 \mathrm{~min}$ after slaughter and $24 \mathrm{~h}$ afterwards. Meat colour was assessed by $L^{*}, a^{*}$ and $b^{*}$ system (CIE, 1986) using a colorimeter Minolta $\mathrm{CR}-10$. The colour was measured on muscle surface immediately after cutting and after $24 \mathrm{~h}$ of exposure to the air under refrigeration.

Cooking loss was evaluated in the refrigerated meat samples of similar geometry, individually placed inside polyethylene bags in a water bath at $75^{\circ} \mathrm{C}$. Samples were heated to an internal temperature of $70^{\circ} \mathrm{C}$ (monitored with thermocouples introduced in the core) and cooled for 15 min under running tap water. They were taken from the bags, dried with filter paper and weighed. Cooking loss was expressed as the percentage loss related to the initial weight. After measurement of cooking loss, samples were stored in a refrigerator and used for objective tenderness determinations (after equilibration at room temperature). The Warner-Bratzler shear force was evaluated in sub-samples (at least 3 ) of $1 \mathrm{~cm}^{2}$ cross section and $3-4 \mathrm{~cm}$ in length with fibres perpendicular to the direction of the blade attached to a Stevens QTS 25 apparatus.

Chemical analyses were determined according to AOAC (1990) methods. Samples were analysed in duplicate for moisture, ash and crude protein $(N \times 6.25)$. Lipid analysis was performed by ether-extraction in a Tecator Soxtec HT 1043 (Höganäs, Sweden) according to the procedure described by Tecator (ASTN, 1988).

\subsection{Statistical analysis}

Data was analysed using the GLM procedures of SAS (2002) to determine the influence of slaughter weight and sex and their interaction on carcass and meat quality characteristics. The interaction effect was excluded because it

Table 1

Means ( \pm standard error) of slaughter live weight (SLW), age at slaughter (AS), hot carcass weight of PDO (HCW-PDO) and cold carcass weight (CCW) of male and female lambs

\begin{tabular}{|c|c|c|c|c|c|c|}
\hline Weight classes $(\mathrm{kg})$ & Sex & $n$ & AS (days) & SLW (kg) & HCW-PDO ${ }^{\mathrm{a}}(\mathrm{kg})$ & $\overline{\mathrm{CCW}(\mathrm{kg})}$ \\
\hline \multirow[t]{2}{*}{$<8$} & $\hat{o}$ & 9 & 20 & $6.9 \pm 0.33$ & $4.5 \pm 0.25$ & $3.5 \pm 0.18$ \\
\hline & 우 & 14 & 20 & $6.5 \pm 0.29$ & $4.2 \pm 0.20$ & $3.2 \pm 0.15$ \\
\hline $8-11$ & $\hat{0}$ & 13 & 34 & $9.7 \pm 0.27$ & $5.9 \pm 0.18$ & $4.8 \pm 0.14$ \\
\hline \multirow[t]{2}{*}{$>11$} & $\hat{0}$ & 7 & 47 & $12.6 \pm 0.61$ & $7.9 \pm 0.39$ & $6.4 \pm 0.34$ \\
\hline & q & 6 & 57 & $12.8 \pm 0.65$ & $7.9 \pm 0.43$ & $6.3 \pm 0.36$ \\
\hline
\end{tabular}

\footnotetext{
${ }^{\text {a }}$ Includes head, liver, lungs, heart and kidneys.
} 
was not significant $(P>0.05)$. Least-square means were computed and tested for differences using Bonferroni test. Using carcass weight as an independent variable $(x, g)$, allometric growth coefficients $(b)$ for each carcass joint and dissected tissue $(y, g)$ were calculated using the Huxley model, $\log y=b \log x+\log a$.

\section{Results and discussion}

The number of carcasses and the means of slaughter live weight, slaughter age and carcass weights in each group are presented in Table 1 .

\subsection{Carcass traits and composition}

Dressing proportion, based on cold carcass weight, and carcass fatness $(\mathrm{KKCF} / \mathrm{CCW})$ were not affected by weight class and sex (Table 2). Previous studies with unweaned lambs demonstrated an increase of dressing percentage and carcass fatness with slaughter weight (Díaz et al., 2005; Velasco et al., 2000). This was not observed, possibly because "Terrincho-DOP" lambs were slaughtered at lower weights or we compared smaller weight ranges. In the present study, the KKCF proportion was similar to that found by Díaz et al. (2005) in Manchego suckling lambs slaughtered at $5.5-6.5 \mathrm{~kg}$ and $>6.5 \mathrm{~kg}$ of $\mathrm{HCW}$ (2.27\% and $2.47 \%$, respectively).

Linear and perimeter measurements and carcass compactness index were not influenced by sex but were affected by slaughter weight (Table 2). Carcass measurements and carcass compactness index increased significantly $(P<$ 0.001 ) with increased live weight. Russo et al. (2003) and Díaz et al. (2005) also found that an increase in carcass weight in light lambs was related to increased compactness.

The effects of sex and slaughter weight on joint percentages are reported in Table 3. With only one exception, there was no gender effect on joint proportions. This result is consistent with those reported by Miguélez, Zumalacárregui, Osorio, Beteta, and Mateo (2006) for PGI suckling lambs, in which gender did not affect the proportion of commercial cuts, except for the neck percentage. Slaughter live weights of $<8 \mathrm{~kg}$ showed higher

Table 2

Least-square means ( \pm standard error) of carcass characteristics of male and female lambs slaughtered at three weight classes

\begin{tabular}{|c|c|c|c|c|c|c|c|}
\hline & \multicolumn{2}{|l|}{$\operatorname{Sex}(\mathrm{S})$} & \multicolumn{3}{|c|}{ Weight classes $(\mathrm{W})$} & \multicolumn{2}{|c|}{ Significance } \\
\hline & Male $(n=28)$ & Female $(n=29)$ & $<8.0(n=23)$ & $8.0-11.0(n=21)$ & $>11.0(n=13)$ & $\mathrm{S}$ & $\mathrm{W}$ \\
\hline \multicolumn{8}{|l|}{ Traits } \\
\hline Cold carcass weight $(\mathrm{CCW}), \mathrm{kg}$ & $4.9 \pm 0.12$ & $4.7 \pm 0.12$ & $3.3 \pm 0.13^{\mathrm{c}}$ & $4.7 \pm 0.13^{\mathrm{b}}$ & $6.3 \pm 0.18^{\mathrm{a}}$ & ns & $* * *$ \\
\hline Dressing percentage (CCW basis), \% & $49.8 \pm 0.50$ & $48.9 \pm 0.52$ & $49.7 \pm 0.56$ & $48.4 \pm 0.57$ & $50.0 \pm 0.75$ & ns & ns \\
\hline $\mathrm{KKCF} / \mathrm{CCW}, \%$ & $2.2 \pm 0.16$ & $2.4 \pm 0.17$ & $2.3 \pm 0.18$ & $2.2 \pm 0.18$ & $2.4 \pm 0.24$ & ns & ns \\
\hline Anterior buttock circumference, $\mathrm{cm}$ & $34.4 \pm 0.50$ & $33.5 \pm 0.51$ & $29.1 \pm 0.55^{\mathrm{c}}$ & $34.3 \pm 0.58^{b}$ & $38.4 \pm 0.73^{\mathrm{a}}$ & $\mathrm{ns}$ & *** \\
\hline Posterior buttock circumference, $\mathrm{cm}$ & $38.9 \pm 0.52$ & $38.0 \pm 0.54$ & $33.3 \pm 0.58^{\mathrm{c}}$ & $38.6 \pm 0.61^{\mathrm{b}}$ & $43.5 \pm 0.77^{\mathrm{a}}$ & ns & *** \\
\hline Carcass internal length $(\mathrm{L}), \mathrm{cm}$ & $41.6 \pm 0.43$ & $41.6 \pm 0.45$ & $37.3 \pm 0.48^{\mathrm{c}}$ & $41.9 \pm 0.51^{\mathrm{b}}$ & $45.7 \pm 0.64^{\mathrm{a}}$ & ns & *** \\
\hline Leg length, $\mathrm{cm}$ & $28.6 \pm 0.23$ & $28.1 \pm 0.24$ & $26.0 \pm 0.26^{\mathrm{c}}$ & $28.4 \pm 0.27^{\mathrm{b}}$ & $30.6 \pm 0.34^{\mathrm{a}}$ & ns & $* * *$ \\
\hline Carcass compactness $(\mathrm{CCW} / \mathrm{L})$ & $11.1 \pm 0.46$ & $10.6 \pm 0.47$ & $8.5 \pm 0.41^{b}$ & $11.2 \pm 0.57^{\mathrm{a}}$ & $12.7 \pm 0.67^{\mathrm{a}}$ & ns & *** \\
\hline
\end{tabular}

Least-square means on the same row within sex or slaughter live weight with different superscript are significantly different $(P<0.05)$; ns, not significant. ${ }^{* * *} P<0.001$.

Table 3

Least-square means ( \pm standard error) of joints percentages of male and female suckling lambs slaughtered at three weight classes

\begin{tabular}{|c|c|c|c|c|c|c|c|}
\hline \multirow[t]{2}{*}{ Joints $(\%)$} & \multicolumn{2}{|l|}{$\operatorname{Sex}(S)$} & \multicolumn{3}{|c|}{ Weight classes (W) } & \multicolumn{2}{|c|}{ Significance } \\
\hline & Male $(n=28)$ & Female $(n=29)$ & $<8.0(n=23)$ & $8.0-11.0(n=21)$ & $>11.0(n=13)$ & $\mathrm{S}$ & W \\
\hline$\overline{\mathrm{Leg}}$ & $27.6 \pm 0.20$ & $27.6 \pm 0.20$ & $28.5 \pm 0.22^{\mathrm{a}}$ & $27.5 \pm 0.23^{\mathrm{b}}$ & $26.8 \pm 0.29^{b}$ & ns & **** \\
\hline Shoulder & $21.6 \pm 0.24$ & $21.7 \pm 0.25$ & $21.3 \pm 0.26$ & $21.9 \pm 0.28$ & $21.6 \pm 0.35$ & ns & ns \\
\hline Loin & $10.4 \pm 0.15$ & $10.3 \pm 0.16$ & $10.4 \pm 0.17$ & $10.1 \pm 0.18$ & $10.6 \pm 0.22$ & ns & ns \\
\hline Rib & $6.5 \pm 0.11^{\mathrm{b}}$ & $6.9 \pm 0.11^{\mathrm{a}}$ & $6.6 \pm 0.12$ & $6.7 \pm 0.12$ & $6.6 \pm 0.15$ & & ns \\
\hline Anterior rib & $5.3 \pm 0.10$ & $5.3 \pm 0.10$ & $5.5 \pm 0.11$ & $5.3 \pm 0.11$ & $5.2 \pm 0.14$ & ns & ns \\
\hline Higher-priced & $46.2 \pm 0.17$ & $46.3 \pm 0.18$ & $46.7 \pm 0.19^{\mathrm{a}}$ & $45.9 \pm 0.20^{\mathrm{b}}$ & $46.1 \pm 0.25^{\mathrm{ab}}$ & ns & $*$ \\
\hline
\end{tabular}

Higher-priced joints: leg + loin + chump; Least-square means on the same row within sex or slaughter live weight with different superscript are significantly different $(P<0.05)$; ns, not significant.

${ }^{*} P<0.05$.

*** $P<0.001$. 
leg, neck and higher-priced cuts (leg + loin + chump) proportions and lower chump proportions (Table 3). However, these differences were small (less than $2 \%$ ) and do not justify any differences in carcass commercial value. The significant decrease in leg proportion was also observed by Kremer et al. (2004), as the carcass weight increased from a minimum of 8 to a maximum of $23.5 \mathrm{~kg}$ in Corriedale lambs. These results reflect differences in the growth pattern of different regions of the carcass (Table 4) and are in agreement with previous findings

Table 4

Growth coefficients $(b)$ and standard error (se) of carcass joints relative to half carcass weight in female and male Borrego Terrincho lambs

\begin{tabular}{|c|c|c|c|c|c|c|}
\hline \multirow[t]{2}{*}{ Carcass joints (g) } & \multicolumn{2}{|c|}{ Female $(n=29)$} & \multicolumn{2}{|c|}{ Male $(n=28)$} & \multicolumn{2}{|c|}{ Pooled data $(n=57)$} \\
\hline & $b$ & se & $b$ & se & $b$ & se \\
\hline Leg & $0.88^{*}$ & 0.02 & $0.93^{* *}$ & 0.03 & $0.90^{* *}$ & 0.02 \\
\hline Shoulder & 0.98 & 0.05 & 1.02 & 0.03 & 1.00 & 0.03 \\
\hline Chump & $1.17^{* *}$ & 0.05 & $1.15^{* *}$ & 0.05 & $1.15^{* *}$ & 0.04 \\
\hline Loin & 1.04 & 0.05 & 1.05 & 0.06 & 1.05 & 0.04 \\
\hline Rib & 0.99 & 0.05 & 1.03 & 0.10 & 1.00 & 0.05 \\
\hline Anterior rib & 0.95 & 0.05 & 0.89 & 0.07 & 0.93 & 0.04 \\
\hline Breast & $1.23^{*}$ & 0.09 & $1.15^{*}$ & 0.06 & $1.21^{* *}$ & 0.05 \\
\hline Neck & 0.96 & 0.05 & $0.84^{*}$ & 0.06 & 0.93 & 0.04 \\
\hline
\end{tabular}

Coefficients on the same line do not differ between sexes $(P>0.05)$.

${ }^{*} b \neq 1$ for $P<0.05$.

** $b \neq 1$ for $P<0.01$.

Table 5

Least-square means ( \pm standard error) of muscle and bone tissues percentages and ratios of male and female suckling lambs slaughtered at three weight classes

\begin{tabular}{|c|c|c|c|c|c|c|c|}
\hline \multirow[t]{2}{*}{ Traits } & \multicolumn{2}{|l|}{ Sex (S) } & \multicolumn{3}{|c|}{ Weight classes $(\mathrm{W})$} & \multicolumn{2}{|c|}{ Significance } \\
\hline & Male $(n=28)$ & Female $(n=29)$ & $<8.0(n=23)$ & $8.0-11.0(n=21)$ & $>11.0(n=13)$ & $S$ & $\mathrm{~W}$ \\
\hline \multicolumn{8}{|c|}{ Muscle (M), \% } \\
\hline Leg & $64.7 \pm 0.30^{\mathrm{b}}$ & $65.6 \pm 0.31^{\mathrm{a}}$ & $64.6 \pm 0.33$ & $65.2 \pm 0.34$ & $65.6 \pm 0.43$ & * & ns \\
\hline Shoulder & $61.5 \pm 0.34$ & $62.4 \pm 0.35$ & $61.8 \pm 0.38$ & $62.7 \pm 0.40$ & $61.2 \pm 0.50$ & ns & ns \\
\hline Chump & $60.1 \pm 0.61^{\mathrm{b}}$ & $62.2 \pm 0.63^{\mathrm{a}}$ & $61.5 \pm 0.68$ & $61.7 \pm 0.71$ & $60.4 \pm 0.90$ & * & ns \\
\hline Loin & $64.5 \pm 0.79^{\mathrm{b}}$ & $66.8 \pm 0.82^{\mathrm{a}}$ & $65.9 \pm 0.88$ & $66.5 \pm 0.92$ & $64.6 \pm 1.16$ & * & ns \\
\hline Rib & $57.9 \pm 0.66$ & $59.4 \pm 0.68$ & $59.5 \pm 0.74$ & $59.0 \pm 0.77$ & $57.4 \pm 0.97$ & ns & ns \\
\hline Anterior rib & $60.6 \pm 0.74$ & $62.5 \pm 0.77$ & $61.7 \pm 0.83$ & $61.5 \pm 0.86$ & $61.3 \pm 1.09$ & ns & ns \\
\hline Breast & $47.9 \pm 0.87$ & $50.7 \pm 0.89$ & $50.7 \pm 0.96$ & $49.5 \pm 1.01$ & $47.7 \pm 1.27$ & ns & ns \\
\hline Neck & $56.5 \pm 0.61^{\mathrm{b}}$ & $58.9 \pm 0.63^{\mathrm{a}}$ & $58.3 \pm 0.68$ & $58.3 \pm 0.72$ & $56.5 \pm 0.90$ & ${ }^{* *}$ & ns \\
\hline Carcass & $60.3 \pm 0.42^{b}$ & $61.9 \pm 0.44^{\mathrm{a}}$ & $61.4 \pm 0.47$ & $61.5 \pm 0.50$ & $60.4 \pm 0.62$ & * & ns \\
\hline \multicolumn{8}{|l|}{ Bone (B), \% } \\
\hline Leg & $24.3 \pm 0.30$ & $23.5 \pm 0.31$ & $26.0 \pm 0.33^{\mathrm{a}}$ & $23.8 \pm 0.35^{\mathrm{b}}$ & $21.8 \pm 0.44^{\mathrm{c}}$ & ns & *** \\
\hline Shoulder & $24.3 \pm 0.26^{\mathrm{a}}$ & $23.4 \pm 0.27^{\mathrm{b}}$ & $26.2 \pm 0.29^{\mathrm{a}}$ & $23.5 \pm 0.30^{\mathrm{b}}$ & $21.9 \pm 0.38^{\mathrm{c}}$ & & *** \\
\hline Chump & $17.6 \pm 0.39$ & $17.1 \pm 0.40$ & $18.9 \pm 0.43^{\mathrm{a}}$ & $16.9 \pm 0.45^{\mathrm{b}}$ & $16.1 \pm 0.57^{\mathrm{b}}$ & ns & ${ }^{* * *}$ \\
\hline Loin & $15.0 \pm 0.47$ & $14.0 \pm 0.48$ & $16.4 \pm 0.52^{\mathrm{a}}$ & $14.5 \pm 0.55^{\mathrm{b}}$ & $12.7 \pm 0.69^{\mathrm{b}}$ & ns & $* * *$ \\
\hline Rib & $21.0 \pm 0.54$ & $20.5 \pm 0.55$ & $22.2 \pm 0.60^{\mathrm{a}}$ & $21.1 \pm 0.62^{\mathrm{ab}}$ & $19.0 \pm 0.79^{\mathrm{b}}$ & ns & *** \\
\hline Anterior rib & $22.5 \pm 0.56$ & $21.5 \pm 0.58$ & $22.3 \pm 0.63$ & $22.4 \pm 0.66$ & $21.4 \pm 0.82$ & ns & ns \\
\hline Breast & $20.0 \pm 0.68$ & $19.9 \pm 0.70$ & $21.2 \pm 0.57$ & $19.3 \pm 0.73$ & $19.2 \pm 0.73$ & ns & ns \\
\hline Neck & $23.5 \pm 0.67$ & $22.3 \pm 0.69$ & $24.8 \pm 0.74^{\mathrm{a}}$ & $23.1 \pm 0.78^{\mathrm{ab}}$ & $20.8 \pm 0.98^{\mathrm{b}}$ & ns & ${ }^{* * *}$ \\
\hline Carcass & $21.9 \pm 0.29$ & $21.1 \pm 0.30$ & $23.4 \pm 0.33^{\mathrm{a}}$ & $21.4 \pm 0.34^{\mathrm{b}}$ & $19.7 \pm 0.43^{\mathrm{c}}$ & $\mathrm{ns}$ & *** \\
\hline \multicolumn{8}{|l|}{$M: B$} \\
\hline Leg & $2.7 \pm 0.04^{\mathrm{b}}$ & $2.8 \pm 0.04^{\mathrm{a}}$ & $2.5 \pm 0.04^{\mathrm{c}}$ & $2.8 \pm 0.04^{\mathrm{b}}$ & $3.0 \pm 0.05^{\mathrm{a}}$ & $* *$ & ${ }^{* * *}$ \\
\hline Shoulder & $2.5 \pm 0.03^{\mathrm{b}}$ & $2.7 \pm 0.03^{\mathrm{a}}$ & $2.4 \pm 0.03^{\mathrm{b}}$ & $2.7 \pm 0.04^{\mathrm{a}}$ & $2.8 \pm 0.05^{\mathrm{a}}$ & $* *$ & *** \\
\hline Chump & $3.5 \pm 0.08$ & $3.7 \pm 0.09$ & $3.3 \pm 0.09^{b}$ & $3.7 \pm 0.10^{\mathrm{a}}$ & $3.8 \pm 0.12^{\mathrm{a}}$ & ns & $* * *$ \\
\hline Loin & $4.5 \pm 0.17$ & $4.9 \pm 0.17$ & $4.1 \pm 0.19^{\mathrm{b}}$ & $4.8 \pm 0.20^{\mathrm{ab}}$ & $5.2 \pm 0.25^{\mathrm{a}}$ & ns & *** \\
\hline Rib & $2.8 \pm 0.08$ & $3.0 \pm 0.09$ & $2.7 \pm 0.09$ & $2.9 \pm 0.10$ & $3.1 \pm 0.12$ & ns & ns \\
\hline Anterior rib & $2.8 \pm 0.10$ & $3.0 \pm 0.10$ & $2.8 \pm 0.11$ & $2.8 \pm 0.11$ & $3.0 \pm 0.14$ & ns & ns \\
\hline Breast & $2.3 \pm 0.07$ & $2.6 \pm 0.07$ & $2.4 \pm 0.07$ & $2.5 \pm 0.08$ & $2.5 \pm 0.10$ & ns & ns \\
\hline Neck & $2.5 \pm 0.08^{b}$ & $2.7 \pm 0.09^{\mathrm{a}}$ & $2.4 \pm 0.09^{\mathrm{b}}$ & $2.6 \pm 0.10^{\mathrm{ab}}$ & $2.8 \pm 0.12^{\mathrm{a}}$ & * & ${ }^{*}$ \\
\hline Carcass & $2.8 \pm 0.04^{\mathrm{b}}$ & $3.0 \pm 0.04^{\mathrm{a}}$ & $2.6 \pm 0.04^{\mathrm{c}}$ & $2.9 \pm 0.05^{\mathrm{b}}$ & $3.1 \pm 0.06^{\mathrm{a}}$ & $* *$ & *** \\
\hline
\end{tabular}

Least-square means on the same row within sex or slaughter live weight with different superscript are significantly different $(P<0.05)$; ns, not significant.

${ }^{*} P<0.05$.

${ }^{* *} P<0.01$.

${ }^{* * *} P<0.001$. 
in sheep (Kempster, Croston, Guy, \& Jones, 1987; Santos, Azevedo, \& Silva, 2000). Allometric growth coefficients of each carcass joint relative to half carcass weight (pooled data) showed that the leg developed earlier relative to carcass weight, chump and breast developed later and the other joints grew at the same rate as the carcass weight (Table 4).

Tissue carcass percentages obtained by dissection of the left half carcass joints are presented in Tables 5 and 6. Female lambs had higher muscle proportion in all carcass joints, but significant differences were only found in leg, chump, loin and neck (Table 5). Male lambs had higher proportions $(P<0.05)$ of subcutaneous fat in chump and neck, but subcutaneous fat in the carcass was not affected by gender (Table 6). No differences were found between sexes in intermuscular fat (Table 6) and bone proportions (Table 5), except for the shoulder joint $(P<0.05)$. Teixeira, Cadavez, Delfa, and Bueno (2004) observed that the carcass joint composition of Churro Galego Bragançano and Suffolk and Merino Precoce crossbred lambs (8$14 \mathrm{~kg}$ of carcass weight) was highly influenced by sex. In our study, the small differences observed among sexes in dissected fat can be explained by the low slaughter age/ weight, more evident differences might be expected at higher slaughter age/weight. Female lambs had a greater muscle/bone ratio in their carcasses than males ( 2.8 versus 3.0). This was due to the fact that females had proportionately more muscle tissue than males (61.9 versus 60.3$)$, while the proportion of bone did not vary significantly between males and females. Guía, Cañeque, and Lauzurica (1985) also reported that at commercial slaughter weights female suckling lambs display greater muscle than bone tissue development.

Subcutaneous tissue distribution was mainly modified by slaughter weight (Table 6). Increased slaughter weight resulted in a higher proportion of subcutaneous fat in all carcass joints $(P<0.001)$, except for the breast and neck joints. Intermuscular fat proportions increased as live weight increased in neck joint $(P<0.001)$ and carcass $(P<0.05)$ (Table 6). Significant differences $(P<0.05)$ in intermuscular fat were only found between live weight groups below $8 \mathrm{~kg}$ and above $11 \mathrm{~kg}$. Subcutaneous fat was the component of dissectible fat that increased most with slaughter weight (Table 6). These findings agree with results of other authors (Díaz et al., 2005; Russo et al., 2003; Sañudo, Alfonso, Sánchez, Delfa, \& Teixeira, 2000) who found the same variations in fat proportion in light lambs as carcass weight increased. Bone proportion decreased $(P<0.001)$ with live weight in all carcass joints, except for the anterior rib and breast (Table 5). Muscle proportion was stable in all carcass joints for the three slaughter weight classes, ranging from $60.4 \%$ to $61.5 \%$ (Table 5). These results are in agreement with those of Díaz et al. (2005) and Rodrigues et al. (2006). The muscle/bone ratio of the carcasses increased as slaughter weight increased, ranging from 2.6 to 3.1 (Table 5). Mahgoub and Lodge (1994) and Díaz et al. (2003) also observed that muscle/bone ratio increases with slaughter weight. In the present study, significant differences in muscle/bone ratios in the carcass joints were observed between the lightest slaughter weight group and those of the other two groups (Table 5). Allometric growth

Table 6

Least-square means $( \pm$ standard error) of dissected fat percentages of the male and female lambs slaughtered at three weight classes

\begin{tabular}{|c|c|c|c|c|c|c|c|}
\hline \multirow[t]{2}{*}{ Traits } & \multicolumn{2}{|l|}{$\operatorname{Sex}(\mathrm{S})$} & \multicolumn{3}{|c|}{ Weight classes (W) } & \multicolumn{2}{|c|}{ Significance } \\
\hline & Male $(n=28)$ & Female $(n=29)$ & $<8.0(n=23)$ & $8.0-11.0(n=21)$ & $>11.0(n=13)$ & $\mathrm{S}$ & $\mathrm{W}$ \\
\hline \multicolumn{8}{|c|}{ Subcutaneous fat (\%) } \\
\hline Leg & $5.3 \pm 0.27$ & $5.2 \pm 0.28$ & $3.9 \pm 0.30^{\mathrm{b}}$ & $5.3 \pm 0.31^{\mathrm{a}}$ & $6.5 \pm 0.40^{\mathrm{a}}$ & ns & $* * *$ \\
\hline Shoulder & $5.6 \pm 0.28$ & $5.5 \pm 0.29$ & $3.8 \pm 0.31^{\mathrm{c}}$ & $5.2 \pm 0.32^{\mathrm{b}}$ & $7.6 \pm 0.41^{\mathrm{a}}$ & $\mathrm{ns}$ & *** \\
\hline Chump & $12.2 \pm 0.60^{\mathrm{a}}$ & $10.4 \pm 0.62^{\mathrm{b}}$ & $9.2 \pm 0.66^{\mathrm{b}}$ & $11.4 \pm 0.70^{\mathrm{ab}}$ & $13.4 \pm 0.87^{\mathrm{a}}$ & * & *** \\
\hline Loin & $9.5 \pm 0.52$ & $8.7 \pm 0.53$ & $7.1 \pm 0.57^{\mathrm{b}}$ & $8.8 \pm 0.60^{\mathrm{b}}$ & $11.4 \pm 0.76^{\mathrm{a}}$ & ns & *** \\
\hline Rib & $6.1 \pm 0.39$ & $6.2 \pm 0.40$ & $4.4 \pm 0.43^{\mathrm{c}}$ & $6.0 \pm 0.45^{\mathrm{b}}$ & $8.1 \pm 0.57^{\mathrm{a}}$ & ns & *** \\
\hline Carcass & $6.6 \pm 0.30$ & $6.1 \pm 0.31$ & $4.7 \pm 0.34^{\mathrm{c}}$ & $6.3 \pm 0.35^{\mathrm{b}}$ & $7.9 \pm 0.44^{\mathrm{a}}$ & ns & $* * *$ \\
\hline \multicolumn{8}{|c|}{ Intermuscular fat (\%) } \\
\hline Leg & $5.7 \pm 0.17$ & $5.7 \pm 0.18$ & $5.5 \pm 0.19$ & $5.7 \pm 0.20$ & $6.0 \pm 0.25$ & ns & $\mathrm{ns}$ \\
\hline Shoulder & $8.6 \pm 0.23$ & $8.7 \pm 0.24$ & $8.2 \pm 0.26$ & $8.6 \pm 0.27$ & $9.3 \pm 0.34$ & ns & $\mathrm{ns}$ \\
\hline Chump & $10.1 \pm 0.29$ & $10.3 \pm 0.30$ & $10.4 \pm 0.32$ & $9.9 \pm 0.33$ & $10.1 \pm 0.42$ & ns & $\mathrm{ns}$ \\
\hline Neck & $15.4 \pm 0.62$ & $15.2 \pm 0.64$ & $13.6 \pm 0.69^{\mathrm{b}}$ & $14.2 \pm 0.72^{\mathrm{b}}$ & $18.1 \pm 0.90^{\mathrm{a}}$ & ns & *** \\
\hline Carcass & $11.2 \pm 0.30$ & $10.9 \pm 0.30$ & $10.5 \pm 0.33^{\mathrm{b}}$ & $10.8 \pm 0.34^{\mathrm{ab}}$ & $11.9 \pm 0.43^{\mathrm{a}}$ & ns & $*$ \\
\hline
\end{tabular}

Least-square means on the same row within sex or slaughter live weight with different superscript are significantly different $(P<0.05)$; ns, not significant.

${ }^{*} P<0.05$.

** $P<0.01$.

${ }^{* * *} P<0.001$. 
coefficients for carcass and joints tissue weights on carcass half weight are shown for male and female lambs, in Table 7. No significant differences $(P>0.05)$ were found in $b$ values between male and female lambs. Muscle grew at a similar rate to the carcass weight in almost of the car-

Table 7

Allometric growth coefficients $(b)$ and standard error (se) of carcass and joints tissue relative to half carcass weight in female and male Borrego Terrincho lambs

\begin{tabular}{|c|c|c|c|c|c|c|}
\hline \multirow[t]{2}{*}{ Tissue joints (g) } & \multicolumn{2}{|c|}{$\begin{array}{l}\text { Female } \\
(n=29)\end{array}$} & \multicolumn{2}{|c|}{$\begin{array}{l}\text { Male } \\
(n=28)\end{array}$} & \multicolumn{2}{|c|}{$\begin{array}{l}\text { Pooled data } \\
(n=57)\end{array}$} \\
\hline & $b$ & se & $b$ & se & $b$ & se \\
\hline \multicolumn{7}{|l|}{ Carcass } \\
\hline Muscle & 0.96 & 0.02 & 1.02 & 0.03 & 0.98 & 0.02 \\
\hline Bone & $0.71^{* *}$ & 0.03 & $0.77^{* *}$ & 0.04 & $0.76^{* *}$ & 0.03 \\
\hline Subcutaneous fat & $2.06^{* *}$ & 0.26 & $1.61^{* *}$ & 0.16 & $1.93^{* *}$ & 0.16 \\
\hline Intermuscular fat & $1.29^{* *}$ & 0.10 & 1.11 & 0.11 & $1.24^{* *}$ & 0.07 \\
\hline \multicolumn{7}{|l|}{ Leg } \\
\hline Muscle & $0.89^{*}$ & 0.03 & 0.99 & 0.03 & $0.92^{* *}$ & 0.02 \\
\hline Bone & $0.59^{* *}$ & 0.04 & $0.70^{* *}$ & 0.05 & $0.65^{* *}$ & 0.03 \\
\hline Subcutaneous fat & $2.00^{* *}$ & 0.23 & $1.49^{* *}$ & 0.17 & $1.83^{* *}$ & 0.15 \\
\hline Intermuscular fat & 1.16 & 0.08 & 0.91 & 0.10 & 1.06 & 0.06 \\
\hline \multicolumn{7}{|l|}{ Shoulder } \\
\hline Muscle & 0.96 & 0.06 & 1.03 & 0.04 & 0.98 & 0.04 \\
\hline Bone & $0.72^{* *}$ & 0.04 & $0.77^{* *}$ & 0.04 & $0.76^{* *}$ & 0.03 \\
\hline Subcutaneous fat & $2.22^{* *}$ & 0.26 & $1.90^{* *}$ & 0.18 & $2.11^{* *}$ & 0.16 \\
\hline Intermuscular fat & $1.20^{* *}$ & 0.11 & $1.19^{* *}$ & 0.10 & $1.19^{* *}$ & 0.07 \\
\hline \multicolumn{7}{|l|}{ Chump } \\
\hline Muscle & $1.13^{*}$ & 0.06 & $1.16^{*}$ & 0.06 & $1.11^{* *}$ & 0.04 \\
\hline Bone & $0.87^{* *}$ & 0.07 & $0.90^{* *}$ & 0.08 & $0.88^{* *}$ & 0.05 \\
\hline Subcutaneous fat & $2.04^{* *}$ & 0.29 & $1.66^{*}$ & 0.24 & $1.95^{* *}$ & 0.19 \\
\hline Intermuscular fat & 1.21 & 0.11 & 1.10 & 0.12 & 1.14 & 0.08 \\
\hline \multicolumn{7}{|l|}{ Loin } \\
\hline Muscle & 1.00 & 0.05 & 1.07 & 0.05 & 1.01 & 0.03 \\
\hline Bone & $0.66^{* *}$ & 0.11 & $0.61^{* *}$ & 0.13 & $0.67^{* *}$ & 0.08 \\
\hline Subcutaneous fat & $1.88^{* *}$ & 0.27 & $1.64^{*}$ & 0.27 & $1.83^{* *}$ & 0.18 \\
\hline Intermuscular fat & 1.27 & 0.19 & 1.10 & 0.17 & 1.23 & 0.13 \\
\hline \multicolumn{7}{|l|}{$R i b$} \\
\hline Muscle & 0.95 & 0.05 & 1.09 & 0.05 & 0.97 & 0.04 \\
\hline Bone & $0.73^{* *}$ & 0.07 & 0.84 & 0.12 & $0.77^{* *}$ & 0.06 \\
\hline Subcutaneous fat & $2.24^{* *}$ & 0.42 & $2.01^{* *}$ & 0.24 & $2.14^{* *}$ & 0.25 \\
\hline Intermuscular fat & 1.23 & 0.17 & 1.04 & 0.31 & 1.19 & 0.16 \\
\hline \multicolumn{7}{|l|}{ Anterior rib } \\
\hline Muscle & 0.97 & 0.06 & 0.92 & 0.08 & 0.94 & 0.05 \\
\hline Bone & $0.76^{* *}$ & 0.08 & 0.86 & 0.14 & $0.82^{* *}$ & 0.07 \\
\hline Intermuscular fat & 1.23 & 0.15 & 0.97 & 0.14 & 1.14 & 0.10 \\
\hline \multicolumn{7}{|l|}{ Breast } \\
\hline Muscle & 1.09 & 0.06 & 1.09 & 0.06 & 1.08 & 0.04 \\
\hline Bone & 1.01 & 0.12 & 1.01 & 0.09 & 1.04 & 0.07 \\
\hline Subcutaneous fat & $2.14^{* *}$ & 0.34 & $1.49^{*}$ & 0.20 & $1.97^{* *}$ & 0.21 \\
\hline Intermuscular fat & 1.36 & 0.28 & $1.34^{*}$ & 0.14 & $1.40^{* *}$ & 0.16 \\
\hline \multicolumn{7}{|l|}{ Neck } \\
\hline Muscle & $0.89^{* *}$ & 0.05 & $0.86^{*}$ & 0.06 & $0.87^{* *}$ & 0.04 \\
\hline Bone & $0.64^{*}$ & 0.10 & $0.64^{* *}$ & 0.13 & $0.68^{* *}$ & 0.08 \\
\hline Subcutaneous fat & $2.26^{* *}$ & 0.39 & 1.18 & 0.27 & $1.99^{* *}$ & 0.26 \\
\hline Intermuscular fat & $1.49^{* *}$ & 0.15 & 1.16 & 0.16 & $1.37^{* *}$ & 0.11 \\
\hline
\end{tabular}

Coefficients on the same line do not differ between sexes $(P>0.05)$.

${ }^{*} b \neq 1$ for $P<0.05$.

** $b \neq 1$ for $P<0.01$. cass joints, but muscle in leg and neck joints grew earlier and muscle in chump grew later. Bone was the most precocious tissue relative to carcass weight $\left(b=0.76^{* *}\right)$, indicating that the bone tissue comprised a diminishing proportion of the carcass as the animals matured. Dissected fat weight was late developing relative to carcass weight, but intermuscular fat of the anterior rib, rib, chump and leg grew at a similar rate to the carcass weight. Dissected muscle, bone and fat grew at different rates in relation to carcass weight (Table 7), which is in agreement with previous studies on sheep (Mahgoub \& Lodge, 1994; Santos et al., 2000). The growth of muscle tissue relative to carcass weight followed the established pattern of growth proposed by Jury, Fourie, and Kirton (1977), who showed that the muscle weight distribution is established immediately after birth and changes little thereafter.

\subsection{Meat quality attributes}

Neither gender nor slaughter weight affected muscle $\mathrm{pH}$ measured $60 \mathrm{~min}$ after slaughter or after $24 \mathrm{~h}$ refrigeration (Table 8). Similarly, Dransfield, Nute, Hogg, and Walters (1990) found no differences in $\mathrm{pH}$ among carcasses of different weights and numerous authors have reported that gender did not significantly influence $\mathrm{pH}$ (Díaz et al., 2003; Horcada et al., 1998; McGeehin, Sheridan, \& Butler, 2001; Vergara \& Gallego, 1999). The pH value $24 \mathrm{~h}$ after slaughter was similar to that of other Portuguese lamb breeds such as Bragançana and Mirandesa (Teixeira et al., 2005) or Spanish suckling lamb breeds of Manchego (Díaz et al., 2005) and Talaverana (Velasco et al., 2000). Muscle colour is extremely important in suckling lambs' production whose carcasses should be pale/pink. In the present study, the lightness values (45.6-47.4) for all the groups are indicative of extremely pale meat. No significant differences were observed between sexes or slaughter weight groups in meat colour parameters (Table 8 ). The non significant differences in meat colour could be explained because both sexes and slaughter weight groups were fed and reared similarly. After $24 \mathrm{~h}$ of storage, meat colour was similar between sexes and weight classes and generally paler (Table 8), indicating a long shelf-life for the products (Russo et al., 2003). Cooking losses and tenderness were not significantly affected by sex or slaughter weight (Table 9). Sañudo et al. (2000) and Díaz et al. (2005) did not find any effect of carcass weight on these parameters. In the present study, shear force increased slightly in the highest group $\left(9.5 \mathrm{~kg} / \mathrm{cm}^{2}\right)$, but not significantly. Intramuscular fat content of LTL was affected by gender $(P<0.05)$ and slaughter weight group $(P<0.01)$, with female lambs and slaughter weight groups $8-11 \mathrm{~kg}$ and $>11 \mathrm{~kg}$ displaying higher proportions of intramuscular fat (Table 7). The chemical composition of the LTL muscle was similar to that found by Russo et al. (2003) in semitendinosus muscle (pelvic limb). According to Wood (1990) 2-3\% intramuscular fat is needed to ensure good organoleptic qualities, 
Table 8

Least-square means ( \pm standard error) of meat $\mathrm{pH}$ and colour of male and female lambs slaughtered at three weight classes

\begin{tabular}{|c|c|c|c|c|c|c|c|}
\hline \multirow[t]{2}{*}{ Traits } & \multicolumn{2}{|l|}{$\operatorname{Sex}(S)$} & \multicolumn{3}{|c|}{ Weight classes (W) } & \multicolumn{2}{|c|}{ Significance } \\
\hline & Male $(n=28)$ & Female $(n=29)$ & $<8.0(n=23)$ & $8.0-11.0(n=21)$ & $>11.0(n=13)$ & $\mathrm{S}$ & $\mathrm{W}$ \\
\hline $\mathrm{pH} 60 \mathrm{~min}$ & $6.59 \pm 0.03$ & $6.60 \pm 0.04$ & $6.52 \pm 0.04$ & $6.64 \pm 0.04$ & $6.64 \pm 0.05$ & ns & ns \\
\hline \multicolumn{8}{|c|}{ Meat colour at $1 \mathrm{~h}$} \\
\hline$L^{*}$ (lightness) & $46.1 \pm 0.55$ & $45.6 \pm 0.57$ & $46.1 \pm 0.62$ & $46.2 \pm 0.65$ & $45.2 \pm 0.81$ & ns & ns \\
\hline$a^{*}$ (redness) & $16.2 \pm 0.46$ & $16.9 \pm 0.47$ & $16.4 \pm 0.51$ & $16.5 \pm 0.53$ & $16.7 \pm 0.67$ & ns & ns \\
\hline$b^{*}$ (yellowness) & $11.3 \pm 0.25$ & $10.9 \pm 0.24$ & $11.4 \pm 0.27$ & $11.0 \pm 0.28$ & $10.9 \pm 0.35$ & ns & ns \\
\hline$L^{*}$ (lightness) & $47.4 \pm 0.48$ & $46.7 \pm 0.49$ & $47.3 \pm 0.53$ & $47.0 \pm 0.56$ & $46.9 \pm 0.70$ & ns & $\mathrm{ns}$ \\
\hline$a^{*}$ (redness) & $16.5 \pm 0.38$ & $17.1 \pm 0.39$ & $16.3 \pm 0.42$ & $17.2 \pm 0.44$ & $16.9 \pm 0.56$ & ns & $\mathrm{ns}$ \\
\hline$b^{*}$ (yellowness) & $13.6 \pm 0.18$ & $13.7 \pm 0.19$ & $13.4 \pm 0.20$ & $13.7 \pm 0.21$ & $13.7 \pm 0.27$ & ns & ns \\
\hline
\end{tabular}

ns, not significant.

Table 9

Least-square means ( \pm standard error) of meat quality traits of male and female lambs slaughtered at three weight classes

\begin{tabular}{|c|c|c|c|c|c|c|c|}
\hline \multirow[t]{2}{*}{ Traits } & \multicolumn{2}{|l|}{ Sex $(S)$} & \multicolumn{3}{|c|}{ Weight classes (W) } & \multicolumn{2}{|c|}{ Significance } \\
\hline & Male $(n=28)$ & Female $(n=29)$ & $<8.0(n=23)$ & $8.0-11.0(n=21)$ & $>11.0(n=13)$ & $\mathrm{S}$ & W \\
\hline Shear force value $\left(\mathrm{kg} / \mathrm{cm}^{2}\right)$ & $9.1 \pm 0.42$ & $9.0 \pm 0.40$ & $8.9 \pm 0.45$ & $8.8 \pm 0.47$ & $9.5 \pm 0.59$ & ns & ns \\
\hline Moisture (\%) & $75.7 \pm 0.15$ & $75.5 \pm 0.15$ & $75.8 \pm 0.16$ & $75.7 \pm 0.17$ & $75.4 \pm 0.22$ & ns & ns \\
\hline Ash (\%) & $1.1 \pm 0.01$ & $1.1 \pm 0.01$ & $1.1 \pm 0.01$ & $1.1 \pm 0.01$ & $1.1 \pm 0.01$ & ns & ns \\
\hline Fat $(\%)$ & $1.9 \pm 0.11^{\mathrm{b}}$ & $2.3 \pm 0.11^{\mathrm{a}}$ & $1.8 \pm 0.12^{\mathrm{b}}$ & $2.2 \pm 0.12^{\mathrm{a}}$ & $2.3 \pm 0.15^{\mathrm{a}}$ & $*$ & $* *$ \\
\hline Protein $(\%)$ & $21.3 \pm 0.10$ & $21.1 \pm 0.11$ & $21.4 \pm 0.11$ & $21.0 \pm 0.12$ & $21.2 \pm 0.15$ & ns & ns \\
\hline
\end{tabular}

Least-square means on the same row within sex or slaughter live weight with different superscript are significantly different $(P<0.05)$; ns, not significant. ${ }^{*} P<0.05$

${ }^{* *} P<0.01$

therefore female lambs and slaughter weight classes 8 $11 \mathrm{~kg}$ and $>11 \mathrm{~kg}$ presented better intramuscular fat distributions.

\section{Conclusion}

Lambs slaughtered at higher live weights produced carcasses with better conformation than those slaughtered at $<8 \mathrm{~kg}$. There were no significant differences between male and female lambs in carcass fatness, conformation and meat colour parameters. Therefore, the general appearance of carcasses and meat of "Borrego Terrincho-PDO" lambs at the moment of purchase and consumption will be similar for both sexes. Meat quality traits were not affected by increased live weight. Lambs slaughtered at weight classes $8-11 \mathrm{~kg}$ and $>11 \mathrm{~kg}$ and females had the minimum intramuscular fat contents required to ensure satisfactory organoleptic qualities of the meat. The present findings do not support the recommendation to use a slaughter weight below $8 \mathrm{~kg}$; however these results should be confirmed by sensory analysis.

\section{Acknowledgement}

This research was supported by the European Union (Project AGRO no. 246).

\section{References}

AOAC (1990). Official Methods of Analysis. 14th ed., Association of Official Analytical Chemists, Washington, DC, USA.

ASTN (1988). Total fat extraction in certain food products according to AOAC. Application Short Note, Tecator.

Cañeque, V., Perez, C., Velasco, S., Díaz, M. T., Lauzurica, S., Alvarez, I., et al. (2004). Carcass and meat quality of light lambs using principal component analysis. Meat Science, 67, 595-605.

CIE (1986). Colorimetry (2nd ed.). CIE Publications No. 15.2. Commission Internationale de l'Eclairage, Vienna.

Díaz, M. T., la Fuente, J., Lauzurica, S., Pérez, C., Velasco, S., Álvarez, I., et al. (2005). Use of carcass weight to classify Manchego sucking lambs and its relation to carcass and meat quality. Animal Science, 80, 61-69.

Díaz, M. T., Velasco, S., Pérez, C., Lauzurica, S., Huidobro, F., \& Cañeque, V. (2003). Physico-chemical characteristics of carcass and meat Manchego-breed suckling lambs slaughtered at different weights. Meat Science, 65, 1247-1255.

Dransfield, E., Nute, G. R., Hogg, B. W., \& Walters, B. R. (1990). Carcass and eating quality of ram, castrated ram and ewe lambs. Animal Production, 50, 291-299.

Fisher, A. V., \& de Boer, H. (1994). The EAAP standard method of sheep carcass assessment and dissection procedures. Livestock Production Science, 38, 149-159.

Guía, E., Cañeque, V., \& Lauzurica, S. (1985). Estudio de la aptitud cárnica de la raza Talaverana. In ITEA, Vol. Extra no. 5, Zaragoza, pp. 256-258.

Horcada, A., Beriain, M. J., Purroy, A., Lizaso, G., \& Chasco, J. (1998). Effect of sex on meat quality of Spanish lamb breeds (Lacha and Rasa Aragonesa). Animal Science, 67, 541-547. 
Jury, K. E., Fourie, P. D., \& Kirton, A. H. (1977). Growth and development of sheep IV. Growth of the musculature. New Zealand Journal of Agricultural Research, 20, 115-121.

Kempster, A. J., Croston, D., Guy, D. R., \& Jones, D. W. (1987). Growth and carcass characteristics of crossbred lambs by ten sire breeds, compared at the same estimated carcass subcutaneous fat proportion. Animal Production, 44, 83-98.

Kremer, R., Barbato, G., Castro, L., Rista, L., Rosés, L., Herrera, V., et al. (2004). Effect of sire breed, year, sex and weight on carcass characteristics of lambs. Small Ruminant Research, 53, 117-124.

Mahgoub, O., \& Lodge, G. A. (1994). Growth and body compositionof Omani local sheep. 1. Live-weight growth and carcass and non-carcass characteristics. Animal Production, 58, 365-372.

McGeehin, B., Sheridan, J. J., \& Butler, F. (2001). Factors affecting the $\mathrm{pH}$ decline in lamb after slaughter. Meat Science, 58, 79-84.

Miguélez, E., Zumalacárregui, J. M., Osorio, M. T., Beteta, O., \& Mateo, J. (2006). Carcass characteristics of suckling lambs protected by the PGI "Lechazo de Castilla y León" European quality label: Effect of breed, sex and carcass weight. Meat Science, 73, 82-89.

Rodrigues, S., Cadavez, V., \& Teixeira, A. (2006). Breed and maturity effects on Churra Galega Bragançana and Suffolk lamb carcass characteristics: Killing-out proportion and composition. Meat Science, 72, 288-293.

Russo, C., Preziuso, G., \& Verità, P. (2003). EU carcass classification system: carcass and meat quality in light lambs. Meat Science, 64, $411-416$.

Santos, V. A. C., Azevedo, J. M. T., \& Silva, S. (2000). Crescimento relativo dos vários componentes corporais de borregos da raça Ile-deFrance. Revista Portuguesa de Zootecnia, VII(1), 29-41.

Santos-Silva, J., Mendes, I. A., \& Bessa, R. J. B. (2002). The effect of genotype, feeding system and slaughter weight on the quality of light lambs 1. Growth, carcass composition and meat quality. Livestock Production Science, 76, 17-25.
Santos-Silva, J., \& Portugal, A. V. (2001). The effect of weight on carcass and meat quality of Serra da Estrela and Merino Branco lambs fattened with dehydrated lucerne. Animal Research, 50, 289-298.

Sañudo, C., Alfonso, M., Sánchez, A., Delfa, R., \& Teixeira, A. (2000). Carcass and meat quality in light lambs from different fat classes in the EU carcass classification system. Meat Science, 56, 89-94.

Sañudo, C., Sanchez, A., \& Alfonso, M. (1998). Small ruminant production systems and factors affecting lamb meat quality. Meat Science, 49, 29-64.

Sañudo, C., Santolaria, M. P., Maria, G., Osorio, M., \& Sierra, L. (1996). Influence of carcass weight on instrumental and sensory meat quality in intensive production systems. Meat Science, 42, 195-202.

SAS (2002). SAS User's Guide Statistics. Cary, NC: SAS Institute Inc. Version 9, Third edition.

Teixeira, A., Batista, S., Delfa, R., \& Cadavez, V. (2005). Lamb meat quality of two breeds with protected origin designation. Influence of breed, sex and live weight. Meat Science, 71, 530-536.

Teixeira, A., Cadavez, V., Delfa, R., \& Bueno, M. S. (2004). Carcass conformation and joints composition of Churra Galega Bragançana and crossbred lambs by Suffolk and Merino Precoce sire breeds. Spanish Journal of Agricultural Research, 2, 217-225.

Velasco, S., Lauzurica, S., Cañeque, V., Pérez, C., Huidobro, F., Manzanares, C., et al. (2000). Carcass and meat quality of Talaverana breed suckling lambs in relation to gender and slaughter weight. Animal Science, 70, 253-263.

Vergara, H., \& Gallego, L. (1999). Effect of type of suckling and length of lactation period on carcass and meat quality in intensive lamb production systems. Meat Science, 53, 211-215.

Wood, J. D. (1990). Consequences for meat quality of reducing carcass fatness. In J. D. Wood \& A. V. Fisher (Eds.), Reducing fatness in meat animals (pp. 344-397). London, UK: Elsevier Applied Science. 